\title{
Numerical Study of Two-Dimensional Viscous, Chemically Reacting Flow
}

\author{
Tae-Hyeong Yi, Dale A. Anderson†, Donald R. Wilson ${ }^{\dagger}$ and Frank K. Lu ${ }^{\dagger}$ \\ University of Texas at Arlington, Arlington, TX 76019, USA
}

\begin{abstract}
A computational fluid dynamics code for two-dimensional, multi-species, laminar NavierStokes equations is developed to simulate a pulsed detonation based propulsion system and to analyze its performance. The stiffness due to coupling the fluid dynamics and the chemical kinetics is properly taken care of by using a time-operator splitting method and a variable coefficient ordinary differential equation solver. A second-order Roe scheme with a minmod limiter is explicitly used for space descretization, while a second-order, two-step Runge-Kutta method is used for time descretization. In space integration, a finite volume method and a cell-centered scheme are employed. The first-order derivatives in the equations of transport properties are discretized by a second-order central finite difference method. Chemical kinetics for hydrogen-air mixture is modeled using GRI-Mech. Code validation and investigation of a pulsed normal detonation wave engine for inviscid and viscous cases are accomplished in this work. The code is ported to a high-performance parallel machine with Message-Passing Interface.
\end{abstract}

\section{Introduction}

$\mathrm{O}$ VER the last decade, considerable attention has been placed on pulsed detonation for propulsion applications due to its potential advantages. The advantages include higher thrust performance, wider operating range, higher thermodynamic efficiency, mechanical simplicity and economical fuel consumption compared to conventional engines such as turbojet, turbofan, ramjet and scramjet. Recently, Wilson et al. ${ }^{1}$ proposed a novel multi-mode pulsed detonation propulsion system for space access or hypersonic flight. The concept consists of four modes, which are an ejector-augmented pulsed detonation rocket, a pulsed normal detonation wave engine, an oblique detonatin wave engine and a pure pulsed detonation rocket. These modes are illustrated in Fig. 1. The main advantage of this engine is that it operates from a takeoff to hypersonic speed through a single flow path, which should substantially reduce the weight and volume of the vehicle. This conceptual engine might be used for a fully reusable launch system for low-cost space access. Since most of the thrust for this concept is generated in the second mode by upstream traveling normal detonation waves in the supersonic combustion chamber, the analysis of the second mode is crucial to the successful operation of the engine. The objective of this work is, therefore, to develop a proper numerical code and to investigate this mode.

Previously, Kim et al. ${ }^{2}$ developed a code for a two-dimensional, inviscid, chemically reacting flow. A thermally and chemically non-equilibrium flow with a two-temperature model and a two-step hydrogen-air reaction model was used to simulate the flow in a pulsed detonation engine. Instead of using a simple twostep reaction model, the present work uses a multi-reaction chemical mechanism. Further, two-dimensional, multi-species, laminar Navier-Stokes equations are employed to observe viscous effects. To decouple the fluid dynamics and the chemical kinetics, time-operator splitting is used to take care of stiffness due to the chemical reactions. A second-order, two-step Runge-Kutta method is used for time integration and the second-order Roe scheme with the minmod limiter is used for space integration. In the second step, a system of ordinary differential equations to obtain the chemical source term is solved by a variable coefficient ordinary differential equation solver. ${ }^{3}$

*Graduate Research Associate, Department of Mechanical and Aerospace Engineering, Student Member AIAA.

${ }^{\dagger}$ Professor, Department of Mechanical and Aerospace Engineering, Assoc. Fellow AIAA. 


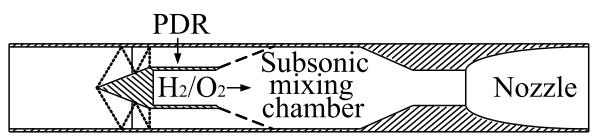

a. Ejector-augmented PDR mode

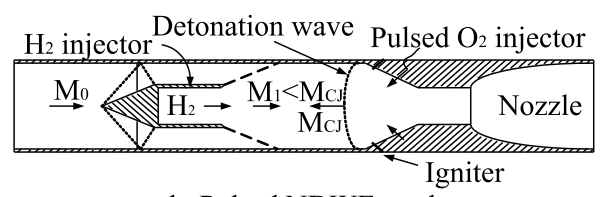

b. Pulsed NDWE mode

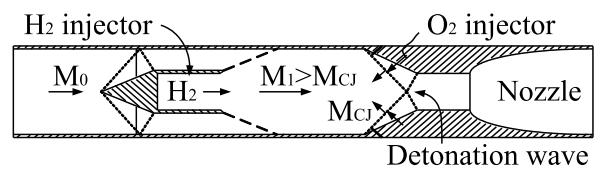

c. Steady ODWE mode

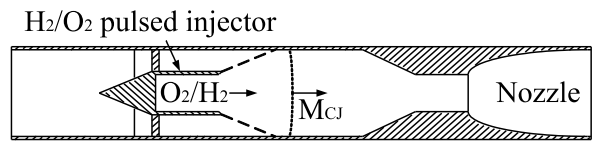

d. Pure PDR mode

Figure 1. Concept of multi-mode pulsed detonation based engine.

Code validation is accomplished for the chemical kinetics and a one-dimensional, chemically reacting detonation tube using a hydrogen-air mixture. The results obtained from these two cases are compared with the Cantera ${ }^{4}$ and $\mathrm{CEA}^{5}$ codes. In the investigation of the second mode of the concept, a non-reacting air flow is first computed up to convergence and then the chemical reactions are initiated by switching the gas to the hydrogen-air mixture. The results of a shock-induced ignition of the detonation are presented for inviscid and viscous cases in the last section.

\section{Governing Equations}

\section{A. Two-Dimensional Navier-Stokes equations}

The governing equations for a two-dimensional, chemically reacting flow that includes transport phenomena such as viscosity, thermal conduction and diffusion are given below. These equations are the two-dimensional, laminar Navier-Stokes equations extended for multi-species flow with chemical reactions: ${ }^{6,7}$

$$
\frac{\partial \vec{Q}}{\partial t}+\frac{\partial \vec{F}}{\partial x}+\frac{\partial \vec{G}}{\partial y}=\vec{S}
$$

where $\vec{Q}, \vec{F}, \vec{G}$ and $\vec{S}$ are defined as

$$
\begin{aligned}
& \vec{Q}=\left[\begin{array}{c}
\rho_{i} \\
\rho u \\
\rho v \\
\rho E
\end{array}\right] \\
& \vec{F}=\vec{F}_{c}-\vec{F}_{v}=\left[\begin{array}{c}
\rho_{i} u \\
\rho u^{2}+p \\
\rho u v \\
(\rho E+p) u
\end{array}\right]-\left[\begin{array}{c}
\rho D_{i, m} \frac{\partial Y_{i}}{\partial x} \\
\tau_{x x} \\
\tau_{x y} \\
u \tau_{x x}+v \tau_{x y}-q_{x}
\end{array}\right] \\
& \vec{G}=\vec{G}_{c}-\vec{G}_{v}=\left[\begin{array}{c}
\rho_{i} v \\
\rho u v \\
\rho v^{2}+p \\
(\rho E+p) v
\end{array}\right]-\left[\begin{array}{c}
\rho D_{i, m} \frac{\partial Y_{i}}{\partial y} \\
\tau_{y x} \\
\tau_{y y} \\
u \tau_{y x}+v \tau_{y y}-q_{y}
\end{array}\right] \\
& \vec{S}=\left[\begin{array}{c}
W_{i} \dot{\omega}_{i} \\
0 \\
0 \\
0
\end{array}\right]
\end{aligned}
$$


where $i=1, \ldots, N_{s} ; N_{s}$ is the number of species, $\rho_{i}$ is the density of species $i, E$ is the total energy, $p$ is the pressure, $u$ and $v$ are the velocity components, $W_{i}$ is the molecular weight of species $i, \dot{\omega}_{i}$ is the mass production rate of species $i$ and $h_{i}$ is the enthalpy of species $i$. The mass fraction of species $i$ is defined as

$$
Y_{i}=\frac{\rho_{i}}{\rho}
$$

In the transport terms in Eq. (2), diffusion due to the pressure and temperature gradients is neglected. The normal and shear stresses can be written in two-dimensional Cartesian coordinates as

$$
\begin{aligned}
\tau_{x x} & =\mu\left(\frac{4}{3} \frac{\partial u}{\partial x}-\frac{2}{3} \frac{\partial v}{\partial y}\right) \\
\tau_{x y} & =\tau_{y x}=\mu\left(\frac{\partial v}{\partial x}+\frac{\partial u}{\partial y}\right) \\
\tau_{y y} & =\mu\left(\frac{4}{3} \frac{\partial v}{\partial y}-\frac{2}{3} \frac{\partial u}{\partial x}\right)
\end{aligned}
$$

The energy fluxes due to thermal conduction and diffusion of species $i$ are given as

$$
\begin{aligned}
& q_{x}=-k \frac{\partial T}{\partial x}-\rho \sum_{i=1}^{N s} h_{i} D_{i, m} \frac{\partial Y_{i}}{\partial x} \\
& q_{y}=-k \frac{\partial T}{\partial y}-\rho \sum_{i=1}^{N s} h_{i} D_{i, m} \frac{\partial Y_{i}}{\partial y}
\end{aligned}
$$

In the above equations, $\mu$ is the mixture viscosity coefficient, $k$ is the mixture thermal conductivity and $D_{i, m}$ is the mass diffusivity of species $i$ to the mixture. From Dalton's law of partial pressures, the pressure of a mixture for a thermally perfect gas is obtained from the summation of the partial pressure of species $i$ :

$$
\begin{gathered}
p=\sum_{i=1}^{N_{s}} p_{i}=\rho R_{i} T \\
\rho=\sum_{i=1}^{N_{s}} \rho_{i} \quad \text { and } \quad R_{i}=\frac{R_{u}}{W_{i}}
\end{gathered}
$$

where $R_{u}$ is universal gas constant.

\section{B. Thermodynamic Relations}

A thermally perfect gas is assumed for each species so that the specific heats, enthalpy and internal energy are functions of temperature only. The specific heat at constant pressure and enthalpy of species $i$ are approximated from least-square coefficients at the given temperature. From Gordon, ${ }^{5}$ therefore, the general forms for $c_{p}^{0}$ and $H^{0}$ are given by

$$
\begin{gathered}
\frac{c_{p}^{0}}{R_{u}}=a_{1} T^{-2}+a_{2} T^{-1}+a_{3}+a_{4} T+a_{5} T^{2}+a_{6} T^{3}+a_{7} T^{4} \\
\frac{H^{0}}{R_{u} T}=-a_{1} T^{-2}+a_{2} T^{-1} \ln T+a_{3}+a_{4} \frac{T}{2}+a_{5} \frac{T^{2}}{3}+a_{6} \frac{T^{3}}{4}+a_{7} \frac{T^{4}}{5}+\frac{a_{8}}{T}
\end{gathered}
$$

where $c_{p}^{0}$ is the molar heat capacity at constant pressure in $\mathrm{J} /(\mathrm{kmol} \cdot \mathrm{K}), H^{0}$ is the molar standard-state enthalpy in $\mathrm{J} / \mathrm{kmol}$ and $a_{n}$ are least-square coefficients. The above equations are further evaluated to convert units to $\mathrm{J} /(\mathrm{kg} \cdot \mathrm{K})$ and $\mathrm{J} / \mathrm{kg}$, respectively:

$$
\begin{gathered}
c_{p i}(T)=\frac{R_{u}}{W_{i}}\left(a_{1} T^{-2}+a_{2} T^{-1}+a_{3}+a_{4} T+a_{5} T^{2}+a_{6} T^{3}+a_{7} T^{4}\right) \\
h_{i}(T)=\frac{R_{u} T}{W_{i}}\left(-a_{1} T^{-2}+a_{2} T^{-1} \ln T+a_{3}+a_{4} \frac{T}{2}+a_{5} \frac{T^{2}}{3}+a_{6} \frac{T^{3}}{4}+a_{7} \frac{T^{4}}{5}+\frac{a_{8}}{T}\right)
\end{gathered}
$$


The specific heat at constant volume and internal energy of speices $i$ are given from $c_{p i}(T)$ and $h_{i}(T)$ as

$$
\begin{gathered}
c_{v i}(T)=c_{p i}(T)-\frac{R_{u}}{W_{i}} \\
e_{i}(T)=h_{i}(T)-\frac{R_{u}}{W_{i}} T
\end{gathered}
$$

The specific heats, specific heat ratio, enthalpy and internal energy of the mixture are given by

$$
\begin{gathered}
c_{p}=\sum_{i=1}^{N_{s}} Y_{i} c_{p i}, \quad c_{v}=\sum_{i=1}^{N_{s}} Y_{i} c_{v i}, \quad \gamma=\frac{c_{p}}{c_{v}} \\
h(T)=\sum_{i=1}^{N_{s}} Y_{i} h_{i}, \quad \text { and } \quad e(T)=\sum_{i=1}^{N_{s}} Y_{i} e_{i}
\end{gathered}
$$

where $Y_{i}$ is the mass fraction of species $i$, which is given as

$$
Y_{i}=\frac{\rho_{i}}{\rho}
$$

It is noted that the specific heat ratio is a function of both the temperature and the density of species $i$ for a mixture of thermally perfect gases. The frozen speed of sound for thermally perfect gases is derived from the thermodynamic relations as ${ }^{6}$

$$
c=\sqrt{\frac{\gamma p}{\rho}}
$$

\section{Transport Properties}

The GRI-Mech transport database ${ }^{8}$ is used to evaluate the viscosity coefficient, the thermal conductivity and the binary diffusion coefficient. The mixture transport properties can be approximated from species properties by mixture-averaged approaches. ${ }^{7}$ The Wilke formula ${ }^{9}$ is used to determine the mixture viscosity coefficient in terms of the mass fractions by

$$
\mu=\sum_{i=1}^{N_{s}} \frac{Y_{i} \mu_{i}}{W_{i}\left(\sum_{j=1}^{N_{s}} \frac{Y_{j} \Phi_{i j}}{W_{j}}\right)}
$$

where

$$
\Phi_{i j}=\frac{1}{\sqrt{8}}\left(1+\frac{W_{i}}{W_{j}}\right)^{-1 / 2}\left[1+\left(\frac{\mu_{i}}{\mu_{j}}\right)^{1 / 2}\left(\frac{W_{j}}{W_{i}}\right)^{1 / 4}\right]^{2}
$$

The mixture thermal conductivity and the mixture-averaged diffusion coefficient of species $i$ are given in terms of the mass fractions, respectively ${ }^{7,10}$

$$
\begin{gathered}
k=\frac{1}{2}\left[W \sum_{i=1}^{N_{s}} \frac{Y_{i} k_{i}}{W_{i}}+\left(W \sum_{i=1}^{N_{s}} \frac{Y_{i}}{W_{i} k_{i}}\right)^{-1}\right] \\
D_{i, m}=\frac{1-Y_{i}}{W\left(\sum_{j \neq i}^{N_{s}} \frac{Y_{j}}{W_{j} D_{j i}}\right)}
\end{gathered}
$$

where the molecular weight of the mixture is defined as

$$
W=\left(\sum_{i=1}^{N_{s}} \frac{Y_{i}}{W_{i}}\right)^{-1}
$$

The species transport properties, that is, $\mu_{i}$ in Eq. (18), $k_{i}$ in Eq. (20) and $D_{j i}$ in Eq. (21) are obtained from classical kinetic theory. For the details, see the reference. ${ }^{7}$ 


\section{Chemical Kinetics}

The overall reaction for the combustion of a stoichiometric hydrogen-air mixture is described as

$$
2 \mathrm{H}_{2}+\mathrm{O}_{2}+3.762 \mathrm{~N}_{2} \rightarrow 2 \mathrm{H}_{2} \mathrm{O}+3.762 \mathrm{~N}_{2}
$$

Two elementary reaction mechanisms for this mixture are extracted from GRI-Mech. ${ }^{8}$ The first mechanism has twenty-eight reversible reactions and nine species $\left(\mathrm{H}_{2}, \mathrm{O}_{2}, \mathrm{~N}_{2}, \mathrm{H}, \mathrm{HO}_{2}, \mathrm{H}_{2} \mathrm{O}, \mathrm{H}_{2} \mathrm{O}_{2}, \mathrm{O}\right.$ and $\left.\mathrm{OH}\right)$ and the second has forty reversible reactions and thirteen species $\left(\mathrm{H}_{2}, \mathrm{O}_{2}, \mathrm{~N}_{2}, \mathrm{H}, \mathrm{HO}_{2}, \mathrm{H}_{2} \mathrm{O}, \mathrm{H}_{2} \mathrm{O}_{2}, \mathrm{~N}, \mathrm{NO}, \mathrm{NO}_{2}\right.$, $\mathrm{N}_{2} \mathrm{O}, \mathrm{O}$ and $\left.\mathrm{OH}\right)$.

From these elementary reactions, the rate of mass production of species $i$ is given by summation over the rate equations of all the elementary reactions $k$ as follows: ${ }^{71}$

$$
\dot{\omega}_{i}=\sum_{k=1}^{N_{r}}\left(v_{i k}^{\prime \prime}-v_{i k}^{\prime}\right)\left[M_{k}\right]\left\{k_{f, k} \prod_{i=1}^{N_{s}}\left[X_{i}\right]^{v_{i k}^{\prime}}-k_{r, k} \prod_{i=1}^{N_{s}}\left[X_{i}\right]^{v_{i k}^{\prime \prime}}\right\}
$$

where $X_{i}$ is the molar concentration of species $i$ and given by $X_{i}=\rho_{i} / W_{i}$. In this equation, the indices $i$ and $k$ denote the number of species and the number of reactions, respectively. $v_{i k}^{\prime}$ indicates stoichiometric coefficients for the reactants, while $v_{i k}^{\prime \prime}$ indicates stoichiometric coefficients for the products. A third body defined below and denoted by $M_{k}$ should appear in Eq. (24) when it is required in some elementary reactions:

$$
\left[M_{k}\right]=\sum_{i=1}^{N_{s}} \alpha_{i k}\left[X_{i}\right]
$$

where $\alpha_{i k}$ is the enhancement factor for species $i$ in the reaction $k$ and given in the chemical mechanism.

The forward rate constant $k_{f, k}$ in reaction $k$ is obtained from a modified three-parameter Arrhenius form ${ }^{7}$

$$
k_{f, k}=A_{k} T^{\beta_{k}} \exp \left(\frac{-E_{k}}{R_{c} T}\right)
$$

where $A_{k}$ is the pre-exponential factor, $\beta_{k}$ is the temperature exponent and $E_{k}$ is the activation energy. The numerical values for these terms are given in a table of the chemical mechanism and obtained from experiment. The universal gas constant $R_{c}$ has the same units as the activation energy $E_{k}$. The reverse rate constant $k_{r, k}$ is determined by the forward rate constant and the equilibrium constant, namely,

$$
k_{r, k}=\frac{k_{f, k}}{K_{c, k}}
$$

The equilibrium constants are calculated from the thermodynamic properties of the species such as

$$
\begin{gathered}
K_{c, k}=K_{p, k}\left(\frac{p_{a t m}}{R_{u} T}\right)^{\sum_{s}}\left(v_{i k}^{\prime \prime}-v_{i k}^{\prime}\right) \\
K_{p, k}=\exp \left[\sum_{i=1}^{N_{s}}\left(v_{i k}^{\prime \prime}-v_{i k}^{\prime}\right)\left(\frac{S_{i}^{0}}{R_{u}}-\frac{H_{i}^{0}}{R_{u} T}\right)\right]
\end{gathered}
$$

where $p_{\text {atm }}$ denotes $p=1 \mathrm{~atm} . S_{i}^{0} / R_{u}$ and $H_{i}^{0} /\left(R_{u} T\right)$ for each species $i$ are obtained from thermodynamic data tables. ${ }^{5}$

\section{Numerical Method}

The governing equations shown in Eq. (1) describe two physical processes, namely, a fluid dynamics and a chemical reaction. In most cases, the chemical reactions have much smaller time scales than those associated with the flow, resulting in stiffness due to the source terms. In order to isolate this stiff source term, time-operator splitting is used in this work. Equation (1) can, therefore, be divided to two steps: (1) 


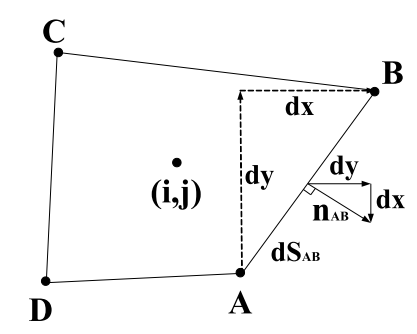

Figure 2. Two-dimensional arbitrary grid cell.

a homogeneous partial differential equation for the fluid dynamics and (2) an ordinary differential equation for chemical reactions: ${ }^{12,13}$

$$
\begin{array}{ll}
\text { 1st step : } & \frac{\partial \vec{Q}}{\partial t}+\frac{\partial \vec{F}}{\partial x}+\frac{\partial \vec{G}}{\partial y}=0, \quad \text { IC }: \vec{Q}^{n} \Rightarrow \vec{Q}^{n+1 / 2} \\
\text { 2nd step }: & \frac{d \vec{Q}}{d t}=\vec{S}, \quad \text { IC }: \vec{Q}^{n+1 / 2} \Rightarrow \vec{Q}^{n+1}
\end{array}
$$

\section{A. Spatial Discretization}

1. Finite Volume Formulation

The set of two-dimensional Navier-Stokes equations given in Eq. (1) can be expressed in integral form without the source term as

$$
\frac{\partial}{\partial t} \int_{\mathcal{V}} \vec{Q} d \mathcal{V}+\oint_{S} \vec{H} \cdot \vec{n} d S=0
$$

where $\vec{n}$ is the normal unit vector and $\vec{H}$ is

$$
\vec{H}=\vec{F} \hat{\imath}+\vec{G} \hat{\jmath}
$$

in Cartesian coordinates. For an arbitrary, two-dimensional grid cell as shown in Fig. 2, the second term in Eq. (31) at the $\overline{A B}$ boundary yields

$$
(\vec{H} \cdot \vec{n} d S)_{A B}=(\vec{F} \hat{\imath}+\vec{G} \hat{\jmath})(d y \hat{\imath}-d x \hat{\jmath})_{A B}
$$

Therefore, the following spatially discretized form of the governing equations without the source term in a two-dimensional Cartesian coordinate system is obtained as

$$
\frac{\partial \vec{Q}}{\partial t} \Delta \mathcal{V}+\sum_{l=1}^{4}(\vec{F} d y-\vec{G} d x)_{l}=0
$$

\section{Second-Order Roe Scheme}

To evaluate the $\vec{F}$ and $\vec{G}$ fluxes at each boundary given in Eq. (34), the second-order Roe scheme ${ }^{14}$ is used. For the one-dimensional case, the flux $\vec{F}$ can be represented by ${ }^{13}$

$$
\vec{F}_{i+1 / 2}=\frac{1}{2}\left[\vec{F}\left(\vec{Q}_{R}\right)+\vec{F}\left(\vec{Q}_{L}\right)-|\hat{A}|_{i+1 / 2}\left(\vec{Q}_{R}-\vec{Q}_{L}\right)\right]
$$


where

$$
\begin{aligned}
& |\hat{A}|_{i+1 / 2}\left(\vec{Q}_{R}-\vec{Q}_{L}\right)=\sum_{n=1}^{3}|\hat{\lambda}|_{i+1 / 2} W_{n} \\
& \hat{\lambda}_{1}=\hat{u}+\hat{c}, \quad \hat{\lambda}_{2}=\hat{u}, \quad \hat{\lambda}_{3}=\hat{u}-\hat{c} \\
& W_{1}=a_{1} \hat{r}_{1}, \quad W_{2}=\sum_{m=2}^{N_{s}+1} a_{m} \hat{r}_{m}, \quad W_{3}=a_{N_{s}+2} \hat{r}_{N_{s}+2} \\
& a_{1}=\frac{\Delta p+\hat{\rho} \hat{c} \Delta u}{2 \hat{c}^{2}}, \quad a_{1+i}=\Delta \rho_{i}-\hat{Y}_{i} \frac{\Delta p}{\hat{c}^{2}}, \quad a_{N_{s}+2}=\frac{\Delta p-\hat{\rho} \hat{c} \Delta u}{2 \hat{c}^{2}}, \quad i=1 \ldots N_{s} \\
& \left.\hat{r}_{1}=\left[\begin{array}{c}
1 \\
\hat{Y}_{1} \\
\vdots \\
\vdots \\
\hat{Y}_{i} \\
\hat{u}+\hat{c} \\
\hat{H}+\hat{u} \hat{c}
\end{array}\right], \quad \begin{array}{ccc}
\vdots & \cdots & 0 \\
0 & \cdots & \vdots \\
\hat{u} & \cdots & 1 \\
\hat{u}^{2}-\frac{\hat{\phi}_{1}}{\hat{\gamma}-1} & \cdots & \hat{u}^{2}-\frac{\hat{\phi}_{N_{s}+1}}{\hat{\gamma}-1}
\end{array}\right], \quad\left[\begin{array}{c}
\hat{Y}_{1} \\
\vdots \\
\vdots \\
\hat{Y_{i}} \\
\hat{u}-\hat{c} \\
\hat{H}-\hat{u} \hat{c}
\end{array}\right]
\end{aligned}
$$

where the subscripts $R$ and $L$ represent the right and left state, $\hat{\lambda}_{n}$ are eigenvalues, $a_{n}$ are wave strengths, $\hat{r}_{n}$ are the right eigenvectors of the $\hat{A}$ matrix and $\left(^{\wedge}\right)$ indicates Roe-averaged quantities. The quantity $\Delta q=q_{R}-q_{L}$, where $q$ is a primitive variable. ${ }^{13,15}$

To obtain second-order accuracy in space, the MUSCL approach is used. This method to generate the second-order approximation uses a piecewise linear extrapolation on each cell. Therefore, the primitive variables of the right and left states at the cell interface are extrapolated by ${ }^{2,16}$

$$
\begin{aligned}
q_{i+1 / 2}^{L} & =q_{i}+\frac{1-k}{4} \overline{\delta^{+}} q_{i-1 / 2}+\frac{1+k}{4} \overline{\delta^{-}} q_{i+1 / 2} \\
q_{i+1 / 2}^{R} & =q_{i+1}-\frac{1+k}{4} \overline{\delta^{+}} q_{i+1 / 2}-\frac{1-k}{4} \overline{\delta^{-}} q_{i+3 / 2}
\end{aligned}
$$

where the parameter $k$ determines the spactial accuracy of the extrapolation. In the above equations, the slopes of the linear variation within the cell have to be limited to prevent nonphysical oscillations around a discontinuity such as shock waves. This is done by limiter functions: ${ }^{2,16}$

$$
\begin{aligned}
& \overline{\delta^{-}} q_{i+1 / 2}=\operatorname{minmod}\left(\delta q_{i+1 / 2}, \omega \delta q_{i-1 / 2}\right) \\
& \overline{\delta^{+}} q_{i+1 / 2}=\operatorname{minmod}\left(\delta q_{i+1 / 2}, \omega \delta q_{i+3 / 2}\right)
\end{aligned}
$$

The minmod limiter function used in this work are:

$$
\operatorname{minmod}(x, y)= \begin{cases}x & \text { if }|x|<|y| \text { and } x y>0 \\ y & \text { if }|x|>|y| \text { and } x y>0 \\ 0 & \text { if } x y<0\end{cases}
$$

with $1 \leq \omega \leq(3-k) /(1-k)$ and $k \neq 1$.

\section{B. Temporal Discretization}

Equation (34) leads to a system of ordinary differential equations in time:

$$
\frac{d \vec{Q}}{d t}=-\frac{1}{\Delta \mathcal{V}} \mathcal{R}
$$

where $\mathcal{V}$ denotes the control volume and $\mathcal{R}$ is the residual, which is the second term of the left side in Eq. (34). To discretize the equation in time, a second-order, two-step Runge-Kutta method is used as shown below:

$$
\begin{aligned}
\vec{Q}^{n+1 / 2} & =\vec{Q}^{n}-\alpha_{1} \frac{\Delta t}{\Delta \mathcal{V}} \mathcal{R}^{n} \\
\vec{Q}^{n+1} & =\vec{Q}^{n+1 / 2}-\frac{\Delta t}{\Delta \mathcal{V}}\left(\alpha_{2} \mathcal{R}^{n+1 / 2}+\beta \mathcal{R}^{n}\right)
\end{aligned}
$$

7 of 11 
where $\alpha_{1}=1, \alpha_{2}=0.5$ and $\beta=0.5$.

\section{Transport Properties}

Since the viscous fluxes $F_{v}$ and $G_{v}$ given in Eq. (2) are evaluated at each face of the control volume, the velocity components $u$ and $v$, the density $\rho$, the species enthalpy $h_{i}$, the mixture viscosity coefficient $\mu$, the mixture thermal conductivity $k$ and the mass diffusivity $D_{i, m}$ of species $i$ are simply averaged at a face. The evaluation of the first-order derivatives of the velocity components, the temperature and the mass fractions is accomplished by the second-order central finite difference method, which requires a transformation from Cartesian coordinates $(x, y)$ to curvilinear coordinates $(\xi, \eta)$.

\section{Evaluation of Source Terms}

The equation of the second step in Eq. (30) is a system of ordinary differential equations since it is a function of the species density only for a given temperature, that is,

$$
\frac{d X_{i}}{d t}=W_{i} \dot{\omega}_{i}
$$

Here, $W_{i}$ is added for converting units from $\mathrm{kmol} /\left(\mathrm{m}^{3} \cdot \mathrm{s}\right)$ to $\mathrm{kg} /\left(\mathrm{m}^{3} \cdot \mathrm{s}\right)$. This system of ordinary differential equations is solved using VODE ${ }^{3}$ which takes care of the stiffness problem arising due to the chemical reactions. Everytime the species density is updated by VODE, the temperature is evaluated with updated values of species density and enthalpy by Newton-Raphson method such as ${ }^{13}$

$$
T^{n+1}=T^{n}-\frac{f(T)}{f^{\prime}(T)}
$$

where

$$
\begin{gathered}
f(T)=\sum_{i=1}^{\mathrm{N}_{\mathrm{s}}} \rho_{i} h_{i}(T)-\rho E+\rho \frac{\overrightarrow{V^{2}}}{2}-T \sum_{i=1}^{\mathrm{N}_{\mathrm{s}}} \rho_{i} R_{i} \\
f^{\prime}(T)=\sum_{i=1}^{\mathrm{N}_{\mathrm{s}}} \rho_{i} c_{p i}(T)-\sum_{i=1}^{\mathrm{N}_{\mathrm{s}}} \rho_{i} R_{i}
\end{gathered}
$$

The iteration is initialized with the temperature of the preceding time step with the density and total energy held constant.

\section{E. Parallel Processing with Message-Passing Interface (MPI)}

The code developed for this work is enabled for parallel processing. A cluster computer system running on MS-Windows server consists of ten processors with gigabit ethernet network. As an implementation of MPI, NT-MPICH is chosen since it shows better performance than MPICH-NT. The code uses one-dimensional Cartesian decomposition to allocate a computational domain to each processor.

\section{Code Validation}

\section{A. Chemical Modeling}

More complex chemical reaction mechanisms are used in this work compared previous work. ${ }^{1,2}$ The chemical mechanism in this case consists of twenty-eight reversible reactions of the hydrogen-air mixture with nine species. The variation of species mole fractions with time is shown in Fig. 3 to validate the chemical mechanism. The same initial mole fractions given in Eq. (23) are used with two conditions, that are, $p=1$ atm, $T=1500 \mathrm{~K}$ and $p=5 \mathrm{~atm}, T=3000 \mathrm{~K}$. The species mole fractions are compared data obtained with Cantera. ${ }^{4}$ The lines in the figures are obtained from the present code and the symbols are obtained from Cantera. As shown in Fig. 3, there is good agreement between the two approaches. The species mole fractions eventually approach to equilibrium states within $10^{-4} \mathrm{~s}$ in Fig. 3(a) and within $10^{-5} \mathrm{~s}$ in Fig. 3(b). Table 1 shows a comparison of the transport properties obtained with $p=5$ atm and $T=3000 \mathrm{~K}$. 


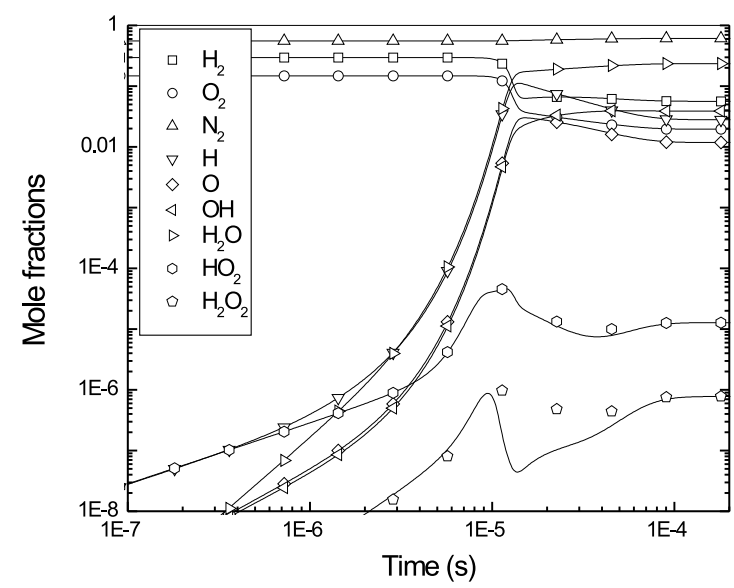

(a) $p=1 \mathrm{~atm}, T=1500 \mathrm{~K}$.

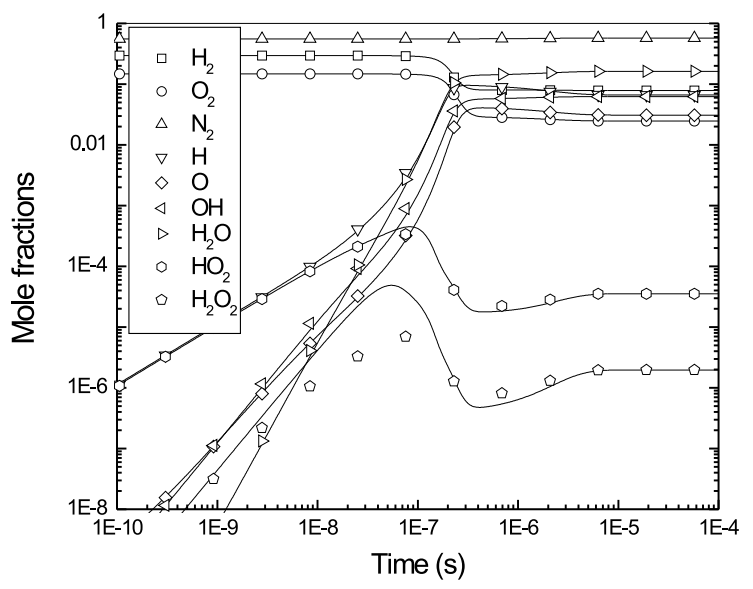

(b) $p=5 \mathrm{~atm}, T=3000 \mathrm{~K}$.

Figure 3. Hydrogen-air reactions at different initial conditions.

Table 1. Comparison of transport properties $(\mathrm{p}=5 \mathrm{~atm}, \mathrm{~T}=3000 \mathrm{~K})$.

\begin{tabular}{|c|c|c|}
\hline Properties & Present code & Cantera code \\
\hline$\mu(\mathrm{Pa} \cdot \mathrm{s})$ & $9.736475 \mathrm{E}-05$ & $9.786610 \mathrm{E}-05$ \\
\hline$k(\mathrm{~W} / \mathrm{m} \cdot \mathrm{K})$ & $3.266989 \mathrm{E}-01$ & $3.253111 \mathrm{E}-01$ \\
\hline$D_{\mathrm{H}_{2}, \mathrm{~m}}\left(\mathrm{~m}^{2} / \mathrm{s}\right)$ & $9.943296 \mathrm{E}-04$ & $9.931041 \mathrm{E}-04$ \\
\hline$D_{\mathrm{O}_{2}, m}$ & $2.808651 \mathrm{E}-04$ & $2.807928 \mathrm{E}-04$ \\
\hline$D_{\mathrm{N}_{2}, m}$ & $2.410256 \mathrm{E}-04$ & $2.412061 \mathrm{E}-04$ \\
\hline$D_{\mathrm{H}, m}$ & $1.634890 \mathrm{E}-03$ & $1.634828 \mathrm{E}-03$ \\
\hline$D_{\mathrm{O}, m}$ & $4.308430 \mathrm{E}-04$ & $4.305356 \mathrm{E}-04$ \\
\hline$D_{\mathrm{OH}, m}$ & $4.230481 \mathrm{E}-04$ & $4.227524 \mathrm{E}-04$ \\
\hline$D_{\mathrm{H}_{2} \mathrm{O}, m}$ & $3.778736 \mathrm{E}-04$ & $3.787855 \mathrm{E}-04$ \\
\hline$D_{\mathrm{HO}_{2}, m}$ & $2.808570 \mathrm{E}-04$ & $2.807522 \mathrm{E}-04$ \\
\hline$D_{\mathrm{H}_{2} \mathrm{O}_{2}, m}$ & $2.791090 \mathrm{E}-04$ & $2.790048 \mathrm{E}-04$ \\
\hline
\end{tabular}

\section{B. One-Dimensional Detonation Tube Simulation}

The one-dimensional chemically reacting flow in a detonation tube is simulated to validate the code. The 0.2 $\mathrm{m}$-long tube is closed at the left end and open at the right end. The entire tube is filled with a stoichiometric mixture of $2 \mathrm{H}_{2}+\mathrm{O}_{2}+3.762 \mathrm{~N}_{2}$ at $p=1 \mathrm{~atm}, T=298.15 \mathrm{~K}$ and $u=0 \mathrm{~m} / \mathrm{s}$. The solid wall boundary condition at the left end and the constant pressure (1 atm) boundary condition at the right end are presented. The fuel mixture is directly ignited at the left end of a tube by an arc where the ignition conditions are numerically given as a pressure of $30 \mathrm{~atm}$ and a temperature of $3000 \mathrm{~K}$.

The results shown in Figs. 4(a)-(c) are the pressure, temperature and density profiles at $t=77.32 \mu \mathrm{s}$. Figures 4(d)-(f) shows mass fractions of $\mathrm{H}_{2}, \mathrm{O}_{2}$ and $\mathrm{H}_{2} \mathrm{O}$ at the same time. The detonation velocities obtained from the CEA code ${ }^{5}$ and the present code were $1975.60 \mathrm{~m} / \mathrm{s}$ and $1968.92 \mathrm{~m} / \mathrm{s}$ and the left wall pressures were $5.91 \mathrm{~atm}$ and $5.88 \mathrm{~atm}$, respectively. From the pressure and temperature obtained at $x=0.05$ $\mathrm{m}$ in Figs. 4(a) and (b), the mass fractions of $\mathrm{H}_{2}, \mathrm{O}_{2}$ and $\mathrm{H}_{2} \mathrm{O}$ were computed by CEA as $0.00132,0.00744$ and 0.239, while they are indicated in Figs. 4(d)-(f) as $0.00142,0.00797$ and 0.238 , respectively. In Fig. 4(b), the temperature near the left end is still high enough to ignite the refilling fuel-air mixture so that a purging process might be required to prevent the preignition. 


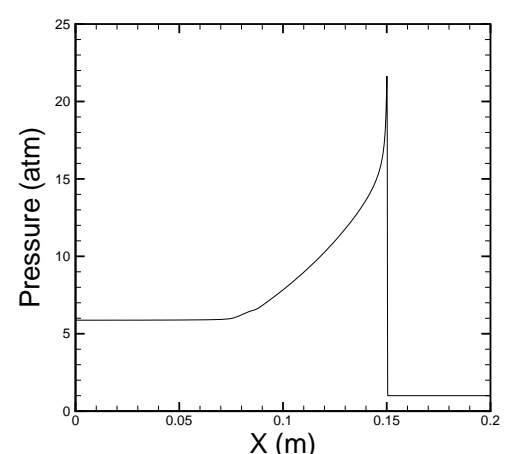

(a) Pressure.

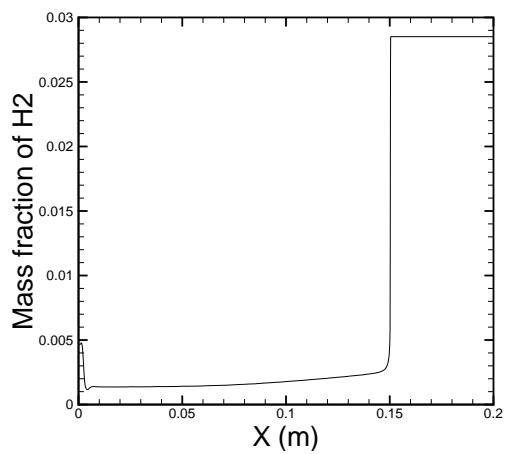

(d) Mass fraction of $\mathrm{H}_{2}$.

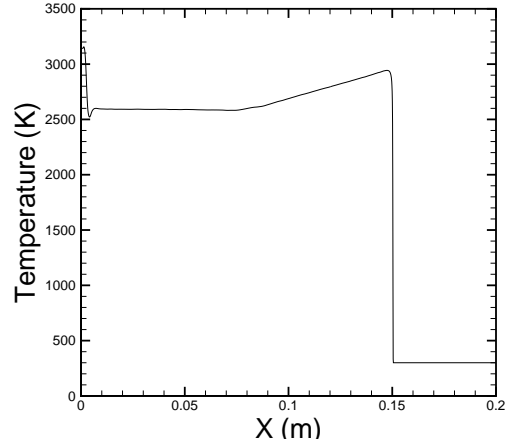

(b) Temperature.

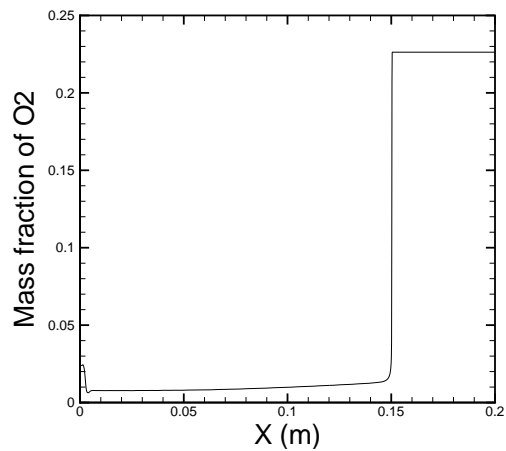

(e) Mass fraction of $\mathrm{O}_{2}$.

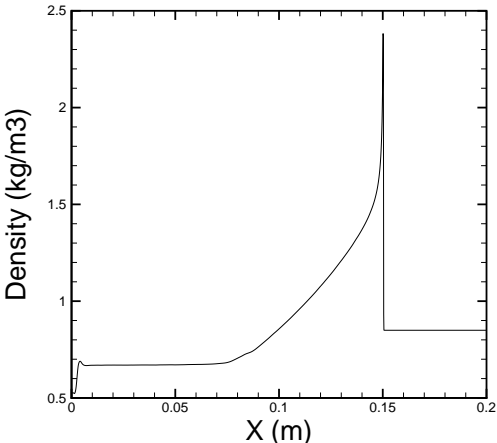

(c) Density.

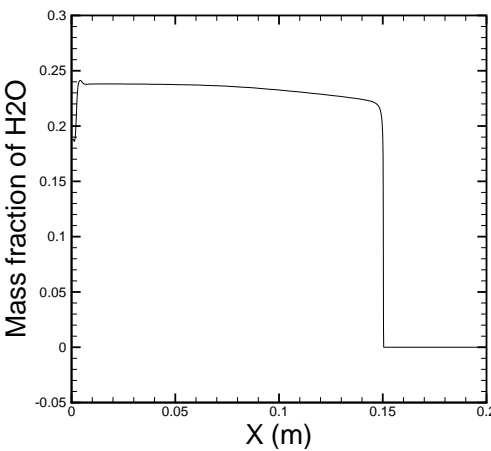

(f) Mass fraction of $\mathrm{H}_{2} \mathrm{O}$.

Figure 4. Profiles of one-dimensional properties in a detonation tube.

\section{Shock-Induced Ignition of Detonation Wave}

In the pulsed normal detonation wave engine mode of the multi-mode PDE concept, the initiation and propagation of the detonation wave in a supersonic combustion chamber are critical issues. For the initiation, the fuel-air mixture could be ignited by an arc or shock waves depending on the incoming flow Mach number and the wedge angle. In this section, shock-induced ignition of the detonation wave is investigated with viscous effects.

Two incoming flow Mach numbers, $M=2.43$ for a viscous flow and $M=4$ for an inviscid flow, at $p=3 \mathrm{~atm}, T=700 \mathrm{~K}$ and a wedge angle of $15^{\circ}$ are used in this case with the stoichiometric hydrogenair mixture. The chemical mechanism is composed of forty reversible elementary reactions with thirteen species. A supersonic inflow at the left side and a constant pressure at the right side are utilized for boundary conditions. Furthermore, non-slip and non-catalytic wall boundary conditions are used in the viscous case where the wall temperature is given as $450 \mathrm{~K}$. An inert air flow is initially simulated using seven species ( $\mathrm{N}, \mathrm{NO}, \mathrm{NO}_{2}, \mathrm{~N}_{2}, \mathrm{~N}_{2} \mathrm{O}, \mathrm{O}$ and $\mathrm{O}_{2}$ ). Once the shock waves are stabilized, the gas is switched to the hydrogen-air mixture using thirteen species.

Figures 5(a) and (b) show pressure distributions after initiating the detonation wave for $M=4$ in the inviscid flow and $M=2.43$ in the viscous flow. As described previously ${ }^{17}$ and shown in Fig. 5(a) for the inviscid flow, ignition occurs at the shock intersection at the centerline. But in the viscous flow with the same initial conditions, it is expected that the mixture is ignited around the wedge corners. In the viscous, Mach number 2.43 case as shown in Fig. 5(b), detonation is initiated just behind the shock reflection on the wall due to the presence of a boundary layer. As the chamber Mach number is increased, the velocity of the detonation moving upstream is decreased and the wave strength is increased. In the inviscid case shown in Fig. $5(\mathrm{a})$, which is for the chamber Mach number of 4 , the rate of propagation of the detonation wave is very low compared to the case in Fig. 5(b). At the moment that the chamber Mach number is just greater than the Chapman-Jouguet (CJ) Mach number, the upstream moving detonation wave is convected downstream. 


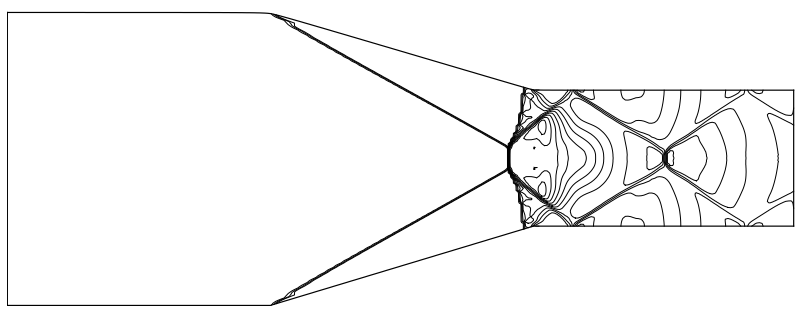

(a) $\mathrm{M}=4$ in an inviscid flow

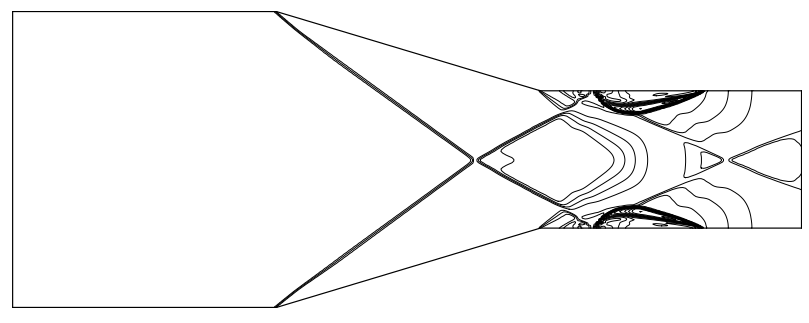

(b) $\mathrm{M}=2.43$ in a viscous flow

Figure 5. Pressure distribution at $\mathrm{p}=3$ atm and $\mathrm{T}=700 \mathrm{~K}$.

\section{Conclusions}

A two-dimensional numerical code for chemically reacting flow is developed to investigate the concept of the multi-mode pulsed detonation based engine. The code uses the multi-reaction chemical mechanisms and includes the transport properties to observe the viscous effects. To evaluate the chemical reactions and the transport properties of the fuel-air mixture, the GRI-Mech database ${ }^{8}$ is used. The VODE code $^{3}$ with the time-operator splitting method is empolyed to take care of stiffness in the present study.

The code was validated in the chemical modeling and one-dimensional, chemical reacting detonation tube cases. The results were compared with the Cantera code ${ }^{4}$ and the CEA code ${ }^{5}$ and showed good agreement with each other. The initiation of the detonation induced by the shock waves in the supersonic wedged combustion chamber was studied in the inviscid and viscous flows for the pulsed normal detonation wave engine mode. For the low incoming flow Mach number, it was observed that the fuel-air mixture was ignited from the hot boundary layer in the viscous flow, while no ignition occurred in the inviscid flow.

\section{References}

${ }^{1}$ Wilson, D.R., Lu, F.K., Kim, H.Y., and Munipalli, R., "Analysis of a Pulsed Normal Detonation Wave Engine Concept," AIAA Paper 2001-1784, 2001.

${ }^{2}$ Kim, H.W., Lu, F.K., Anderson, D.A., and Wilson, D.R., "Numerical Simulation of Detonation Process in a Tube," Computational Fluid Dynamics Journal, Vol. 12, No. 2, 2003, pp. 227-241.

${ }^{3}$ Brown, P.N., Byrne, G.D., and Hindmarsh, A.C., "VODE: A Variable Coefficient ODE Solver," SIAM Journal on Scientific and Statistical Computing, Vol. 10, 1989, pp. 1038-1051. (http://www.1lnl.gov/CASC/).

${ }^{4}$ Goodwin, D.G., Cantera: Object-Oriented Software for Reacting Flows, (http://www. cantera.org).

${ }^{5}$ Gordon, S. and McBride B.J., "Computer Program for Calculation of Complex Chemical Equilibrium Compositions and Applications I. Analysis," NASA RP-1311, 1976 (http://www.lerc.nasa.gov/WWW/CEAWeb/).

${ }^{6}$ Anderson, J.D., Hypersonic and High Temperature Gasdynamics, McGraw-Hill, New York, 1998.

${ }^{7}$ Kee, R.J., Coltrin, M.E., and Glarborg, P., Chemically Reacting Flow: Theory and Practice, Wiley-Interscience, 2003.

${ }^{8}$ Smith, G.P., Golden, D.M., Frenklach, M., Moriarty, N.W., Eiteneer, B., Goldenberg, M., et al., GRI-Mech 3.0, (http://www.me.berkeley.edu/gri_mech/).

${ }^{9}$ Wilke, C.R., "A Viscosity Equation for Gas Mixtures," Chemical Physics, Vol. 18, No. 4, 1950, pp. 517-519.

${ }^{10}$ Fedkiw, R.P., V., Merriman, B., and Osher, S., "High Accurary Numerical Methods for Thermally Perfect Gas Flows with Chemistry," Journal of Computational Physics, Vol. 132, 1997.

${ }^{11}$ Turns, S.R., An Introduction to Combustion: Concepts and Applications, McGraw-Hill, New York, 1996.

${ }^{12}$ Leveque, R.J., Finite Volume Methods for Hyperbolic Problems, Cambridge University Press, 2002.

${ }^{13}$ Deiterding, R., "Parallel Adaptive Simulation of Multi-Dimensional Detonation Structures," Ph.D. dissertation, Brandenburgische Technische Universität Cottbus, Germany, 2003.

${ }^{14}$ Roe, P.L., "Approximate Riemann Solvers, Parameter Vectors and Difference Schemes," Journal of Compututational Physics, Vol. 43, No. 2, 1981, pp. 357-372.

${ }^{15}$ Grossmann, B. and Cinella, P., "Flux-Split Algorithms for Flows with Nonequilibrium Chemistry and Vibrational Relaxation," Journal of Computational Physics, Vol. 88, 1990, pp. 131-168.

${ }^{16}$ Tannehill, J.C., Anderson, D.A., and Pletcher, R.H., Computational Fluid Mechanics and Heat Transfer, 2nd ed., Taylor and Francis, Washington, D.C., 1997.

${ }^{17}$ Lu, F.K., Fan, H.Y., and Wilson, D.R., "Propulsion Utilizing Detonation Waves Induced by a Confined Wedge," ISABE2005-1173, 2005. 